\title{
Gemeinsam mobil machen - Laufen für die Patientensicherheit
}

\author{
Nadja Jennia, Olga Frank ${ }^{b}$, Christoph Bosshard ${ }^{c}$, Esther Kraft ${ }^{d}$

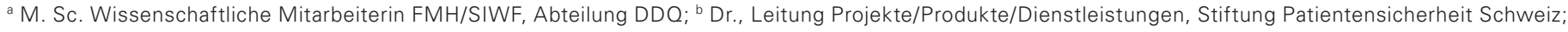 \\ ${ }^{\circ}$ Dr. med., Mitglied des Zentralvorstandes der FMH, Departementsverantwortlicher DDQ; dic. rer. oec., Leiterin Abteilung DDQ
}

\author{
Weil Patientensicherheit alle angeht, veranstaltet die Stiftung «Patientensicherheit \\ Schweiz» gemeinsam mit ihren Partnern - die FMH gehört selbstredend dazu - vom \\ 14. bis 18. September 2015 eine Aktionswoche.
}

Für die Förderung der Patientensicherheit in der Schweiz wird viel getan. Unermüdlich arbeiten die Ärztinnen und Ärzte und alle weiteren Gesundheitsfachpersonen an der Verbesserung der Behandlungsqualität und setzen sich für die Patientensicherheit ein. Unterstützt werden sie dabei von «Patientensicherheit Schweiz», einer Stiftung, die das Thema auf nationaler Ebene bearbeitet und ihm so höchste Priorität einräumt.

Weil Patientensicherheit alle angeht, veranstaltet die Stiftung gemeinsam mit ihren Partnern - die FMH gehört selbstredend dazu - vom 14. bis 18. September 2015 die Aktionswoche «Patientensicherheit - mit Patientinnen und Patienten im Dialog». Die Aktionswoche setzt sich folgende Ziele:

- Durchführung öffentlichkeitswirksamer Aktionen durch die Stiftung selbst und in Kooperation mit den Partnerorganisationen der Stiftung und Gesundheitsinstitutionen;

- Sensibilisierung der Öffentlichkeit zum Thema Patientensicherheit und deren gesundheitspolitischer Bedeutung;

- Die Aktionswoche bietet den Gesundheitseinrichtungen eine Plattform, um ihre Anstrengungen für die verbesserte Patientensicherheit intern und extern darzustellen;

- Die Stiftung für Patientensicherheit ist national bekannt und wird als Kompetenzstelle für die Patientensicherheit in der Schweiz wahrgenommen.

Korrespondenz:

FMH

Nadja Jenni

Elfenstrasse 18

CH-3000 Bern 15

Tel. 0313591111

Fax 0313591112

nadja.jenni[at]fmh.ch
Die FMH nutzt die Aktionswoche, um die Öffentlichkeit zu sensibilisieren und auch um ihre eigenen Anstrengungen darzustellen. In Zusammenarbeit mit der Stiftung lanciert die FMH verschiedene Aktivitäten. Die Mitglieder der FMH werden über die Aktionswoche und deren Inhalte informiert, anlässlich der Aktions- woche wird die Schweizerische Ärztezeitung zum Themenschwerpunkt Patientensicherheit erscheinen und am 14. September 2015 soll unter dem Motto «Gemeinsam mobil machen - Laufen für die Patientensicherheit!» auf dem Waisenhausplatz in Bern ein Spendenlauf stattfinden. Ziel der Veranstaltung ist es, die breite Öffentlichkeit mit verschiedenen Aktionen auf Massnahmen aufmerksam zu machen, die zu einer hohen Patientensicherheit beitragen.

Die FMH nutzt die Aktionswoche, um die Öffentlichkeit zu sensibilisieren und ihre eigenen Anstrengungen darzustellen.

Die Idee ist, diesen Spendenlauf mittels einer Sammelkampagne (Crowdfunding) zu finanzieren. Alle - ob Privatpersonen, Leistungserbringer, Institutionen usw. - können Runden kaufen und diese selber laufen oder laufen lassen. Die Teilnahmebedingungen und weitere Informationen stehen auf der Website der FMH zur Verfügung.

Gemeinsam machen wir mobil! Mit jeder Runde wird die Bevölkerung auf die Patientensicherheit aufmerksam gemacht. Mit jeder Runde steigt die Aufmerksamkeit, mit jeder Runde wird Geld gesammelt, das die Stiftung Patientensicherheit Schweiz zweckgebunden in die Patientensicherheit investiert. Die Stiftung Patientensicherheit Schweiz wird zusammen mit der FMH ein Projekt festlegen und dieses anlässlich des Spendenlaufs präsentieren. Helfen Sie mit und unterstützen Sie den Spendenlauf - damit die sichere medizinische Versorgung auch weiterhin gefördert werden kann! Bis zum 30. Juni 2015 können Sie Runden kaufen. Nähere Informationen sowie die Teilnahmebedingungen finden Sie auf der Website der FMH. 